\title{
Convulsive Movements in Bilateral Paramedian Thalamic and Midbrain Infarction
}

\author{
Kazuo Yamashiro ${ }^{a}$ Tsuyoshi Furuya $^{a}$ Kazuyuki Noda ${ }^{a}$ \\ Takao Urabe $^{b}$ Nobutaka Hattori ${ }^{b}$ Yasuyuki Okuma ${ }^{a}$ \\ ${ }^{a}$ Department of Neurology, Juntendo University Shizuoka Hospital, Shizuoka, \\ and ${ }^{b}$ Juntendo University School of Medicine, Tokyo, Japan
}

\section{Key Words}

Cerebral infarction · Convulsive movements · Seizures · Mesencephalic reticular formation

\begin{abstract}
Although some previous reports have described convulsive movements in bilateral paramedian thalamic and midbrain infarction, little is known about their nature. A 71-yearold man presented with impaired consciousness and clonic movements of both arms. Each series of movements lasted 10 to $20 \mathrm{~s}$ and occurred at 2- to 3-min intervals, which disappeared after intravenous administration of diazepam and phenytoin. Magnetic resonance imaging showed acute bilateral paramedian thalamic and midbrain infarction. A review of the literature revealed that convulsive movements were observed mostly at the onset of infarction. Clonic movements appeared frequently in the limbs, particularly in both arms. Clinical observations and results of animal experiments suggest that these seizures might originate from the mesencephalic reticular formation. Physicians should recognize this condition, because not only seizure control but also early management of ischemic stroke is required.
\end{abstract}

\section{Introduction}

Lesions in the thalamus and those extending into the midbrain can cause various movement disorders such as dystonia, asterixis, and ballism-chorea. Dysfunction of the neural networks involving the thalamus may contribute to these abnormal movements [1]. Convulsive movements have been reported in bilateral paramedian thalamic and midbrain infarction [2-7]. However, little is known about their nature. We here report on the case of a patient with clonic movements of both arms that were observed at the onset of infarction and offer a review of the literature. 


\section{Case Report}

A 71-year-old man, with a ten-year history of chronic hemodialysis due to diabetic nephropathy, was brought to the emergency room. His wife found him unconscious beside his bed. On admission, his blood pressure was 185/86 mm Hg and his pulse was regular. He was in a coma but breathing spontaneously. His pupils were $4 \mathrm{~mm}$ in diameter and unreactive to light. There were neither voluntary eye movements nor eye movements in response to oculocephalic reflex. There was no response to plantar stimulation. Spontaneous symmetrical clonic movements of both arms lasting for 10 to $20 \mathrm{~s}$ were observed at 2- to 3-min intervals. The abnormal movements disappeared after intravenous administration of diazepam and phenytoin. The results of laboratory tests on the following were normal: blood cell count, electrolyte level, glucose level, arterial blood gas, and cerebrospinal fluid. Electrocardiography results were normal. Brain magnetic resonance imaging (MRI) showed acute bilateral paramedian thalamic and midbrain infarction (fig. 1). MR angiography showed atherosclerotic changes in the internal carotid arteries and basilar artery. He was treated with argatroban. Electroencephalography (EEG) on the next day showed diffuse slowing. Ten days after admission, he opened his eyes following painful stimulation. He moved his limbs spontaneously and the horizontal oculocephalic reflex was present. He was transferred to a rehabilitation hospital 17 days after admission.

\section{Discussion}

We report on the case of a patient with clonic movements of both arms at the onset of bilateral paramedian thalamic and midbrain infarction. In the literature [2-7], convulsive movements were observed mostly at the onset of infarction. Clonic movements appear frequently in the limbs, particularly in both arms (table 1).

Lesions in the thalamus and those extending into the midbrain can cause various movement disorders such as dystonia, asterixis, and ballism-chorea. The thalamus relays inputs from peripheral sensory pathways, the basal ganglia, and the cerebellum to the cerebral cortex. Lesions in specific thalamic nuclei and dysfunction of the networks may contribute to these abnormal movements [1]. However, dystonia, asterixis, and ballism-chorea are unlikely because of the clonic nature of the movements in our patient.

Although the cerebral cortex is considered important for the development of seizures in general [8], previous experimental studies have suggested that electrical or chemical stimulation of the brainstem tegmentum, particularly the mesencephalic reticular formation (RF), induces convulsive seizures [9-12]. Autopsy and MRI show the involvement of the midbrain tegmentum in patients with convulsive movements [2-7]. However, to the best of our knowledge, such movements have never been observed in bilateral paramedian thalamic infarction without midbrain lesions. These findings suggest that the mesencephalic RF has a crucial role in the generation of convulsive seizures in bilateral paramedian thalamic and midbrain infarction.

Convulsive seizures induced by electrical stimulation of the mesencephalic RF are not eliminated by precollicular transection $[10,11]$, but pontine tegmentum lesions attenuate the convulsions of brainstem origin [13]. These findings suggest that the generation of the motor component in convulsive seizures is induced by the activation of the descending RF. Furthermore, seizures can take a variety of motor patterns depending on the intensity of stimulation. A high intensity of stimulation causes tonic movements, whereas a low intensity of stimulation causes clonic movements of the forelimbs, and the subsequent increases in stimulation intensity produce a spread of 
clonic movements down to include the hind limbs [14]. These motor patterns are also observed following spinal cord stimulation in decapitated animals [15]. It is hypothesized that the rostrocaudal spread observed in convulsions reflects the rostrocaudal spread of discharge down the polysynaptic descending pathways within the intermediate zone of the spinal gray matter [15]. This can explain why clonic movements were sometimes observed only in both arms, but have never been observed in the legs without the involvement of the arms in bilateral paramedian thalamic and midbrain infarction.

In summary, convulsive movements, particulary clonic movements of both arms, can develop in bilateral paramedian thalamic and midbrain infarction as an initial symptom. Physicians should recognize this condition, because not only seizure control but also early management of ischemic stroke is required.

Table 1. Convulsive movements in bilateral paramedian thalamic and midbrain infarction

\begin{tabular}{|c|c|c|c|c|c|}
\hline \multicolumn{2}{|l|}{ Reference } & Sex/age & Time & Movements & Method \\
\hline \multicolumn{2}{|l|}{ Segarra [3] } & M/51 years & Onset & Convulsive seizures & Autopsy \\
\hline Castaigne [4] & Patient 3 & M/51 years & Onset & Clonic movements of both arms & Autopsy \\
\hline \multirow{2}{*}{\multicolumn{2}{|c|}{ Ropper [2] }} & M/29 years & Onset & Clonic limb movements & MRI \\
\hline & & & 2 weeks & $\begin{array}{l}\text { Tonic-clonic right arm shaking followed by } \\
\text { tonic extension of the right arm and leg }\end{array}$ & \\
\hline \multicolumn{2}{|l|}{ Matheus [5] } & M/50 years & Early & Tonic-clonic movements in all limbs & MRI \\
\hline \multirow[t]{2}{*}{ Naganuma [6] } & Patient 1 & M/76 years & Onset & Clonic movements of both arms & MRI \\
\hline & Patient 2 & M/70 years & Onset & Generalized clonic seizure & MRI \\
\hline \multicolumn{2}{|l|}{ Bain [7] } & M/2 days & Onset & Right-hand sided clonic movement & MRI \\
\hline \multicolumn{2}{|l|}{ Our case } & M/71 years & Onset & Clonic movements of both arms & MRI \\
\hline
\end{tabular}



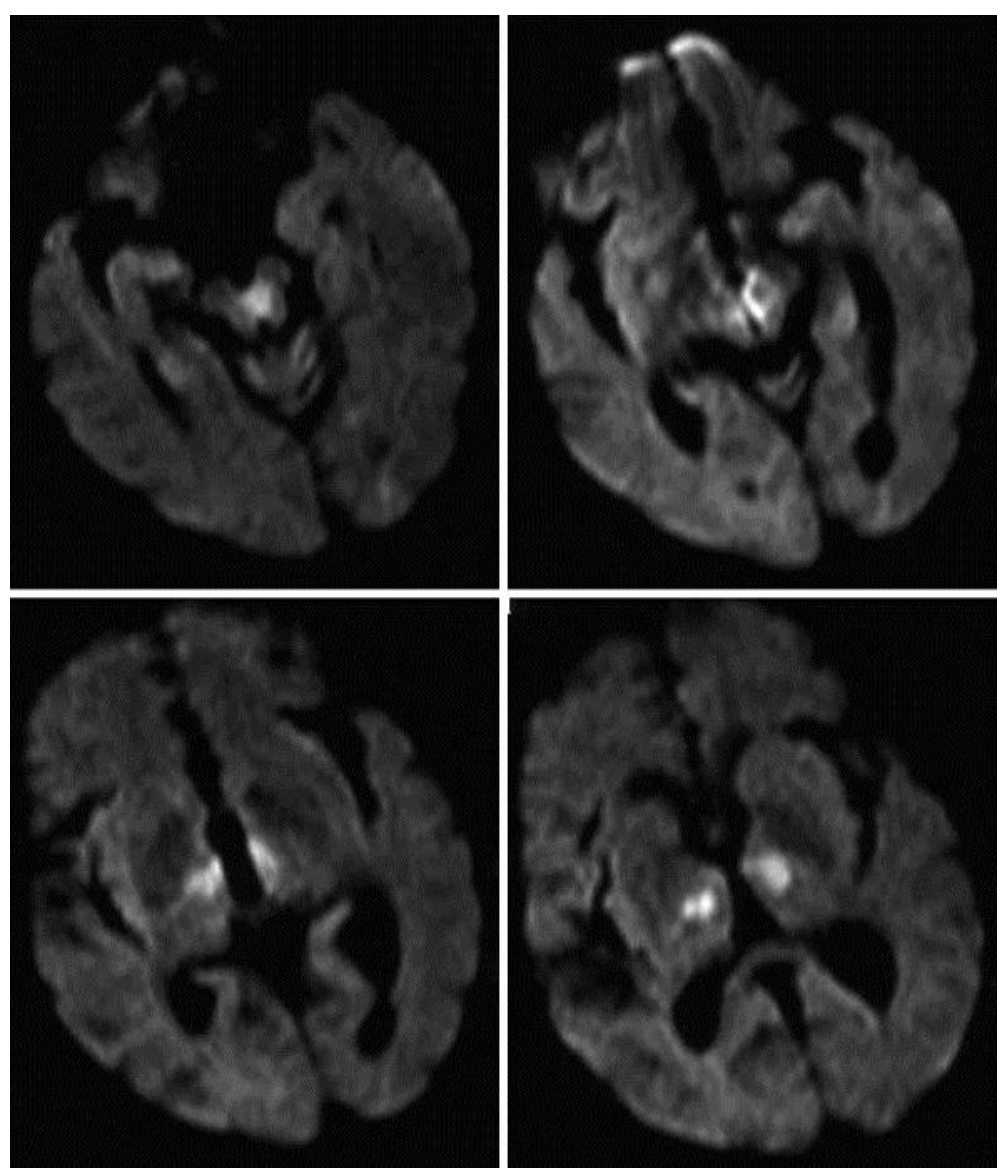

Fig. 1. Diffusion-weighted magnetic resonance imaging demonstrating acute bilateral paramedian thalamic and midbrain infarction.

\section{References}

$>1$ Lee MS, Marsden CD: Movement disorders following lesions of the thalamus or subthalamic region. Mov Disord 1994;9:493-507.

$>2$ Ropper AH: 'Convulsions' in basilar artery occlusion. Neurology 1988;38:1500-1501.

-3 Segarra JM: Cerebral vascular disease and behavior. I. The syndrome of the mesencephalic artery (basilar artery bifurcation). Arch Neurol 1970;22:408-418.

4 Castaigne P, Lhermitte F, Buge A, Escourolle R, Hauw JJ, Lyon-Caen O: Paramedian thalamic and midbrain infarcts: clinical and neuropathological study. Ann Neurol 1981;10:127-148.

5 Matheus MG, Castillo M: Imaging of acute bilateral paramedian thalamic and mesencephalic infarcts. Am J Neuroradiol 2003;24:2005-2008.

6 Naganuma M, Hashimoto Y, Matsuura Y, Terasaki T, Hirano T, Uchino M: Two cases of top of the basilar syndrome with onset seizure. Clin Neurol 2005;45:647-651.

7 Bain SE, Hsieh DT, Vezina LG, Chang T: Bilateral paramedian thalamic and mesencephalic infarcts in a newborn due to occlusion of the artery of Percheron. J Child Neurol 2009;24:219-223.

8 Bladin CF, Alexandrov AV, Bellavance A, Bornstein N, Chambers B, Coté R, Lebrun L, Pirisi A, Norris JW: Seizures after stroke: a prospective multicenter study. Arch Neurol 2000;57:1617-1622.

9 Faingold CL: The role of the brain stem in generalized epileptic seizures. Metab Brain Dis 1987;2:81112. 
10 Kreindler A, Zuckermann E, Steriade M, Chimion D, Kreindler A: Electroclinical features of convulsions induced by stimulation of brain stem. J Neurophysiol 1958;21:430-436.

11 Bergmann F, Costin A, Gutman J: A low threshold convulsive area in the rabbit's mesencephalon. Electroenceph Clin Neurophysiol 1963;15:683-690.

12 Hashizume K, Tanaka T, Fujita T, Tanaka S: Generalized seizures induced by an epileptic focus in the mesencephalic reticular formation: impact on the understanding of the generalizing mechanism. Stereotact Funct Neurosurg 2000;74:153-160.

13 Browning RA: Role of the brain-stem reticular formation in tonic-clonic seizures: lesion and pharmacological studies. Fed Proc 1985;44:2425-2431.

14 Burham WM: Core mechanisms in generalized convulsions. Fed Proc 1985;44:2442-2445.

15 Esplin DW: Spinal cord convulsions. Arch Neurol 1959;1:485-490. 